\title{
SIMULATION MODELING AND OPTIMIZATION USING PROMODEL
}

\author{
Deborah L. Heflin \\ PROMODEL Corporation \\ 1875 South State Street \\ Suite 3400 \\ Orem, UT 84097, U.S.A.
}

\author{
Charles R. Harrell \\ Brigham Young University \\ Manufacturing Engineering \\ $435 \mathrm{CTB}$ \\ Provo, UT 84602, U.S.A.
}

\begin{abstract}
The ProModel Optimization Suite is a powerful yet easyto-use simulation tool for modeling all types of manufacturing systems ranging from small job shops and machining cells to large mass production, flexible manufacturing systems, and supply chain systems. ProModel is a Windows based system with an intuitive graphical interface and object-oriented modeling constructs that eliminate the need for programming. It combines the flexibility of a general-purpose simulation language with the convenience of a data-driven simulator. In addition, ProModel utilizes an optimization tool called SimRunner that performs sophisticated "what-if' analysis by running automatic factorial design of experiments on the model, providing the best answer possible. This tutorial provides an overview of the ProModel Optimization Suite and presents its modeling, analysis, and optimization capabilities.
\end{abstract}

\section{OVERVIEW OF PROMODEL}

ProModel is a simulation and animation tool designed to quickly yet accurately model manufacturing systems of all types, particularly supply chain systems. Engineers and managers find the manufacturing oriented modeling elements and rule-based decision logic extremely easy to learn and use. Users are particularly delighted when they discover that ProModel is capable of modeling their most complex systems. Because it provides such an intuitive and straightforward approach to modeling, it is also attractive to professors in engineering and business programs who are interested in teaching modeling and analysis concepts without having to teach computer programming.

While most systems can be modeled by selecting from ProModel's complete set of modeling elements (e.g. resources, downtimes, etc.) and modifying the appropriate parameters, complete programming capability is also provided if needed for modeling special situations. Built-in language features include if-then-else logic, Boolean expressions, variables, attributes, arrays and even access to external spreadsheet and text files.

For those who prefer coding complex logic using a programming language such as $\mathrm{C}$, Pascal or Basic, external subroutines may be dynamically linked to the model and called from anywhere inside the model at runtime. In this way, ProModel allows systems analysts and simulation experts to use the tools they are most comfortable with to provide total flexibility.

ProModel also provides several built-in distribution functions, which, in conjunction with strewns, return random values according to a statistical distribution. To aid the user in selecting an appropriate distribution for a data set, Stat::Fit is included with ProModel. Stat::Fit is a curve fitting software that fits analytical distributions to user data.

Model development is completely graphical and object-oriented. To the extent possible, all input is provided graphically with information being grouped by object type and presented in a "spreadsheet-like" format for quick and intuitive access. For example, when you define a machine you can define its icon, capacity, downtime characteristics, input and output rules, desired output statistics, etc. ProModel complies with GUI standards, which means that individuals familiar with other standard Windows programs such as word processing or spreadsheets will have no trouble learning how to use ProModel. This data input approach minimizes the learning curve for beginners and maximizes the efficiency for modifying large and complex models.

A unique feature in ProModel is the ability to bring up a pop-up menu depending on the current context that prompts the user in defining any statement or expression. This enables any expression or statement to be entered using only the mouse. It also eliminates the need to remember variable or other element names that you wish to reference by allowing you to select them from a list box.

Quick and convenient online documentation is available through ProModel's integrated Help system and online tutorials. The Help system uses the Windows Help 


\section{Heflin and Harrell}

system that allows maximum flexibility for looking up anything from command syntax to descriptions of model building modules. ProModel also provides tutorials that contain quick lessons on how to build models, how to run models and access output reports and how to model various applications with the software.

To further reduce model development time, ProModel provides model merging capabilities to allow several individuals to be working separately on different sections of a large model. Additionally, frequently defined cells or even commonly used decision logic may be stored as submodel templates which eliminate the need to "re-invent the wheel" with every model. These templates can even have specially designated parameters that may be changed by the user.

Animation development is integrated with the model definition. A major drawback of many simulation software products is that animation development is independent from simulation model development. This makes it time consuming and inconvenient for engineers to use animation as a validation/verification tool. ProModel integrates system definition and animation development into one function. While defining routing locations, conveyors, AGV paths, etc., you essentially develop the animation layout. The layout screen is a virtual screen that can be scaled to an actual factory layout.

Simulation results are informative and may be displayed in tabular or graphical form. Many simulation software products require special commands to generate statistics that are difficult to interpret for nonsimulationists. ProModel allows quick and convenient selection of reports, and provides automatic tabular and graphical reports on all system performance measures. Output reports from several simulation runs can even be compared on the same graph.

ProModel runs on any standard 486 computer with Windows 3.x, Windows NT or Windows 95 operating systems. Most engineers, managers, and professors have easy access to IBM or compatible computers with VGA graphics capabilities. ProModel does not require any special graphics cards or special monitors. This makes it convenient and cost effective for companies and academic institutions that have standard microcomputers. ProModel also runs on LANs (Local Area Networks).

\section{MODELING ELEMENTS}

The modeling elements of ProModel provide the building blocks for representing the physical and logical components of the system being modeled. Physical elements of the system such as parts, machines, or resources may be referenced either graphically or by name. Names of modeling elements may be any word containing up to 80 alphanumeric characters. Following is a brief description of each of these elements (see Figure 1).

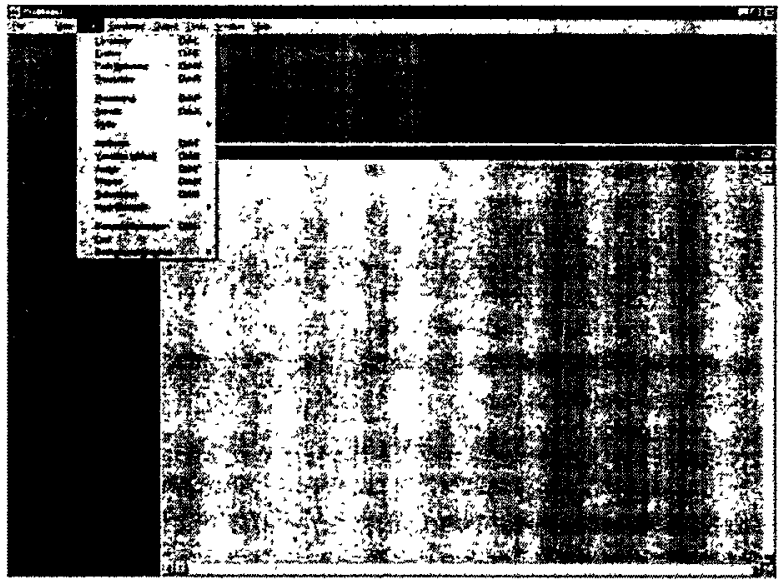

Figure 1: ProModel's Modeling Elements

\subsection{Locations}

Routing locations are fixed places in the system (e.g., machines, queues, storage areas, work stations, tanks, etc.) to where parts or entities are routed for processing, storage or simply to make some decision about further routing. Routing locations may be either single unit locations (e.g. a single machine) or multi-unit locations (e.g. a group of similar machines performing the same operation in parallel).

Routing locations may have a capacity greater than one and may have periodic downtimes as a function of clock time (e.g. shift changes), usage time (e.g. tool wear), usage frequency (e.g. change a dispenser after every $n$ cycles), change of material (e.g. machine setup) or based on some user defined condition. Routing locations may be assigned input and output rules. Input rules are used for selecting what entity to process next while output rules are used for ranking entities (i.e. FIFO, LIFO, user-defined) in a multi-capacity location.

Two special types of locations that provide movement as well as performing holding and operation functions are queues and conveyors. A queue mimics the behavior of waiting lines, including the movement of entities through the line. Conveyors are accumulating or non- accumulating and have a particular speed and load spacing. Conveyors may be configured together to provide conveyor networks.

New to ProModel 4.1 is the ability to schedule independent arrivals. This ability makes creating appointments easy, which can automatically repeated weekly or daily. ProModel can now optimize shift and arrival patterns. Resources can be grouped and statistics collected on the group. ProModel also has an on-screen 24-hour clock and these can be accessed several new functions. 


\subsection{Entities (or parts)}

Parts or entities refer to the items being processed in the system. These include raw materials, piece parts, assemblies, loads, WIP, finished products, etc. Entities of the same type or of different types may be consolidated into a single entity, separated into two or more additional entities or converted to one or more new entity types.

Entities may be assigned attributes that can be tested in making decisions or for gathering specialized statistics. The graphic of an entity can be changed as a result of an operation to show the physical change during the animation.

\subsection{Path Networks}

Path networks are optional and define the possible paths that entities and resources may travel when moving through the system. Path networks consist of nodes connected by path segments and are defined graphically with simple mouse clicks. Multiple path networks may be defined and one or more resources and/or entities may share the same network. Movement along a path network may be defined in terms of distance and speed or by time. Path distances are automatically computed based on the layout scale defined by the user.

There are three types of path networks: passing, nonpassing, and crane. A passing network is used for open path movement where entities and resources are free to overtake one another. Non-passing networks consist of single-file tracks or guide paths such as those used for AGVs where vehicles are not able to pass. Crane networks define the operating envelope and interface points for bridge cranes.

\subsection{Resources}

A resource may be a person, tool, vehicle or other object that may be used to:

- Transport material between routing locations.

- Perform an operation on material at a location

- Perform maintenance on a location or other resource that is down.

Resources may be either static or assigned to a path network for dynamic movement. A special type of dynamic resource is a crane. Built-in decision rules can be used for allocation of resources and for prioritization of part pick-up and delivery. Motion characteristics of resources such as empty and full speeds, acceleration, deceleration, pickup and delivery time can also be specified.

\subsection{Processing (or routing)}

This element defines the processing sequence and flow logic of entities between routing locations. The operation or service times at locations, resource requirements; processing logic, input/output relationship, routing conditions, and move times or requirements can be described using the Processing element.

Operation times can be defined by constants, distributions, functions, attributes, subroutines, etc. or an expression containing any combination of these. Operation logic can include IF-THEN-ELSE statements, loops, nested statement blocks and subroutine calls. Resource related statements such as GET, USE, and JOINTLY GET with Boolean expressions and built-in operation statements such as ACCUM, JOIN, and GROUP greatly simplify otherwise complex logic in describing the processing requirements. Built-in and user-defined routing rules provide flexibility for modeling all types of routing conditions.

\subsection{Arrivals (or production schedule)}

Deterministic, conditional, or stochastic arrivals can be modeled using this element. Independent scheduled arrivals simulate scheduling of repeated appointments. Production schedules can be using independent arrivals. External files including production schedules or arrival data can be read into ProModel in the Arrivals element. Built-in or user de- fined distributions or spreadsheet created data can be used to define inter arrival times and quantities.

\subsection{Shifts (or work schedules)}

A powerful feature is the ability to define custom work and break schedules through ProModel's Shifts module. Work and break schedules are defined graphically by time of day and day of the week. Resources or locations are then assigned to a specific shift schedule. In addition, you can define shift and break logic that controls location and resource behavior when they go off-line and what happens once they are off-line.

\section{ADDITIONAL MODELING ELEMENTS}

ProModel provides additional modeling elements used in statements and expressions to define special decision and operating logic in a model. These elements include variables, attributes, functions, user-defined distributions, and costing.

There are several types of logic elements that may be defined by the user. Like model elements, names given. to these elements may be up to 80 characters in length. Figure 2 shows the menu for accessing these additional elements, some of which will be discussed below. 


\section{Heflin and Harrell}

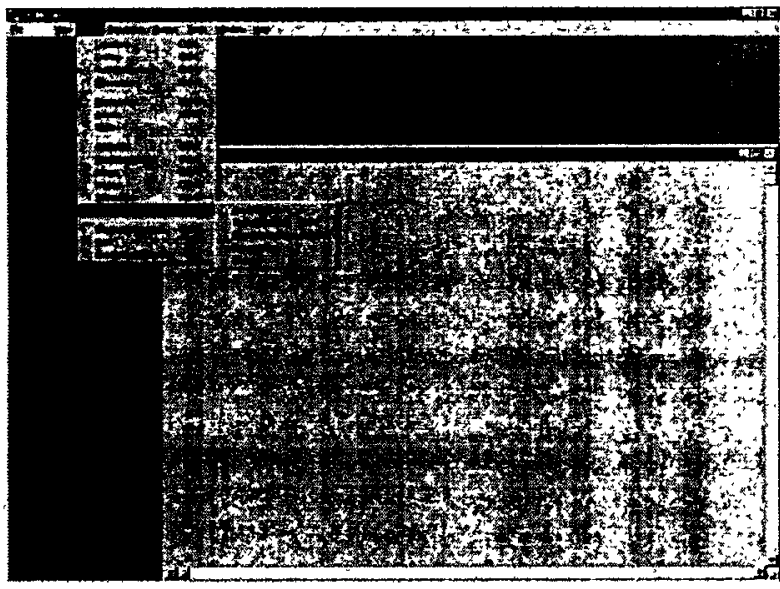

Figure 2: More Elements Menu

\subsection{Attributes}

Attributes for entities and locations can be defined. They may contain real or integer values. Location, resource and entity names may alșo be assigned to attributes.

\subsection{Variables}

Variables are used for decision making and statistical reporting. The value of a variable may be monitored over time and displayed at the end of the simulation as a time series plot or histogram. Variables may hold integer or real values. Local variables may also be used for quick convenience when defining logic.

\subsection{Arrays}

An array is a matrix of variables representing multiple values and may be one-/multi-dimensional.

\subsection{Macros}

A macro is a complex expression or set of expressions that can be defined once and used multiple times as part of a logic statement (i.e. processing, scheduling, downtime logic). Macros are helpful when the same bit of logic is repeated in many places in the model.

\subsection{Subroutines}

A subroutine is a user-defined block of statements that can be passed values when called and optionally return values when completed. Subroutines may be referenced in the place of any value or logic. A complex operation performed several places in a model may be appropriately defined by a single subroutine.

\subsection{Arrival Cycles, Table Functions and User Defined Distributions}

Arrival patterns, table functions or user distributions may be defined. For example, a user defined distribution which returns an operation time of 5 minutes 30 percent of the time and an operation time of 8 minutes 70 percent of the time can be expressed in the User Distributions element.

\subsection{External Files}

One of the powerful features of ProModel is the ability to read data from external text or spreadsheet files or write data to external files. For example, operation times (even in the form of expressions) from an EXCEL spreadsheet file can be read into ProModel using this element.

\section{COSTING}

With ProModel's new Costing feature, you can now make decisions about your system on a cost basis. New dialogs allow you to monitor costs associated with locations, entities, and resources during a model run. In addition, the General Statistics Report now includes Costing statistics, automatically generated at runtime. For locations, you can find the cost of operating a location. For resources, the total cost includes the usage cost as well as the cost of not using the resource. For entities, the total cost includes the costs incurred at all locations the entity passed through, the costs incurred by use of resources, and the initial cost of the entity.

\section{GRAPHICS}

Graphics in ProModel are realistic and easy to createVisually realistic animation helps simulation to become an effective communication vehicle between engineers and managers. ProModel comes with an extensive library of graphics with provision to create and add other graphics to the library. ProModel's Graphic Editor comes with a complete set of drawing tools and a full spectrum of color selections. Scaling, rotating, copying and many other editing features are available. You can even import drawings from other graphics packages. With little effort you can develop quick and simple 2D layouts, or, with little extra effort, 3D perspective layouts. CAD layout drawings (e.g. AutoCAD) can also be brought in to use as the model background. The Graphic Editor is shown in figure 3 . 


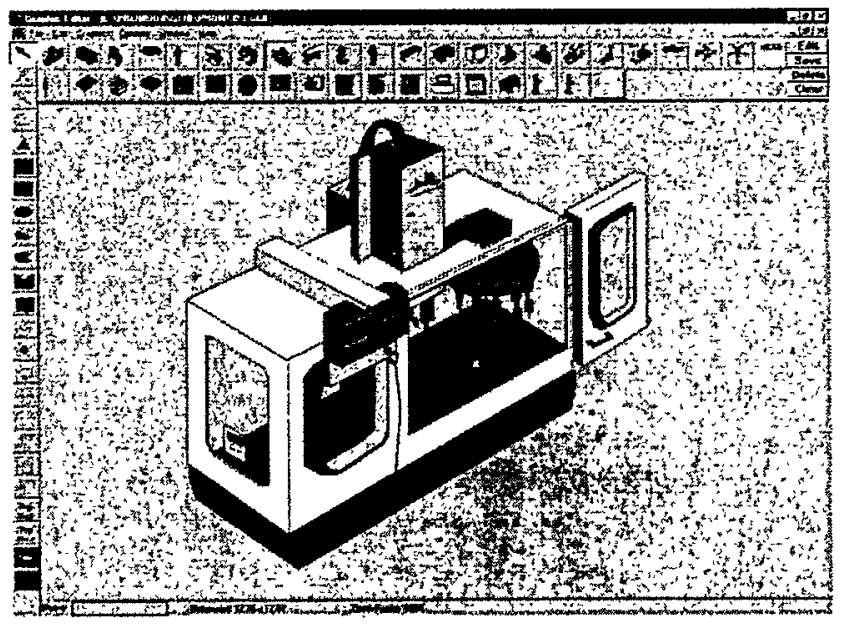

Figure 3: ProModel's Graphic Editor

\section{SCENARIOS AND RUNTIME INTERFACE}

The runtime interface (RTI) is a convenient and controlled environment for modifying selected model parameters (capacities, operation times, etc.) without having to change the model data directly. It also provides an experimental environment, which permits multiple scenarios to be defined and simulated. The RTI can be accessed at the beginning of a simulation run for making modifications for a single run or for saving alternative modifications as scenarios for doing multiple scenario analysis.

\section{RUNNING THE SIMULATION \& ANIMATION}

Models can be run for specified lengths of time or until all entities have been processed. Multiple replications may also be specified. Models may be run with or without animation. The animation is very smooth and it maintains great resolution at any zoom factor.

The Views feature allows you to define and then quickly and easily access specific areas of the model layout. Selecting a view scrolls the layout 'Window and adjusts the zoom so you see a specific region of the layout regardless of the layout window's size. Once a view is defined, you can select it while running the simulation or change the view from within the logic.

During the simulation, you can query the status of resources or the current value of any logic element. Source level debugging allows you to trace each action with the option to display the actual source statement that triggered the action. Trace statements may be confined to a single area of a model. Status lights for locations change colors to help you see various states such as busy, idle, down, etc.

The animation screen is a virtual screen that means the animation layout is limited only by memory in your computer. By turning off the animation, you can speed up the simulation, run for a while and turn the animation back on. The simulation clock resolution can be expressed in terms of hours, minutes, or seconds with a clock resolution of $.0000 \mathrm{I}$ a second.

\section{OUTPUT REPORTS}

By choosing the statistics for resources, locations, entities, variables, etc., users can customize the output reports. The statistics are written to a results database which can be exported to spreadsheets. Additionally, graphical reports of the outputs can be displayed, printed or plotted. These graphs can be individual or comparative pie charts, histograms, time-series plots, etc. See Figure 4 for a sample location state graph.

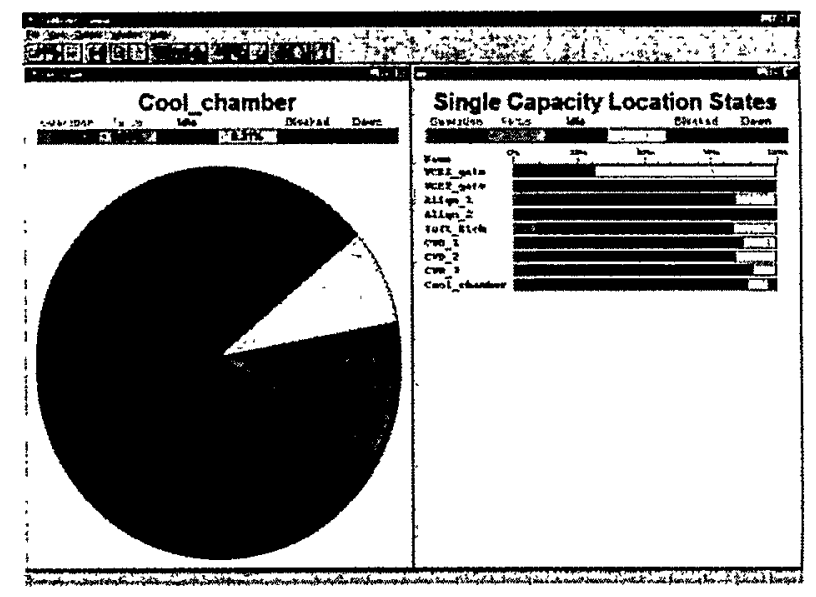

Figure 4: A Location State Graph

\section{OPTIONS}

One of the most accommodating features of ProModel is the provision for customizing the modeling environment to suit the preferences of the user. Under the View menu, you can zoom in or out, display a grid, refresh the screen, set default directories for model files, icon library files, or model output files. You can set and save default settings (i.e. window sizes, appearance).

ProModel also allows you to choose editing preferences for model development. Default fonts and sizes for text and a choice of long or abbreviated menu lengths are available. Prompts and added explanations can also be activated which may assist the novice user. The expert user can benefit from choosing options that streamline the editing process.

\section{OPTIMIZATION}

SimRunner Optimization is an add-on capability for ProModel simulation software. SimRunner turns your simulation model into an answer machine by giving you 


\section{Heflin and Harrell}

the best answer possible while saving you the most time. The optimization process takes an existing ProModel simulation model and performs sophisticated "what-if" analysis and optimization automatically.

For each optimization project, you tell SimRunner which model to analyze/optimize, which input factors to change, and how you measure system simulation performance. You can run two types of projects in SimRunner: Stage One Optimization Analysis, and Stage Two Simulation Optimization.

A Stage One Optimization Analysis is a factorial design of experiments that tells you whether or not changing a given input factor is affecting the objective function, and the significance of the effects. A Stage Two Simulation Optimization is a multi-variable optimization that tries various combinations of input factors to arrive at the combination that provides the best objective function value.

The SimRunner Output report generates three types of data and reporting for you:

1. Data reports for import into spreadsheets

2. Analysis reports for text and word processing reports

3. Charts for graphical display of results. Each of these can be printed or exported to other applications using common clipboard functions.

\section{SUPPLY CHAIN APPLICATIONS}

Simulation models have had extreme value in many companies, being used as decision making and planning tools by managers in supply chain related areas. ProModel is successful in providing an accurate representation for many players involved in a single product line. The players may consist of suppliers, warehouses, customers, retailers, and transportation vehicles. Such issues facing the players of the organization are fleet sizing, inventory reduction, and shutdowns and outages. ProModel allows you to create a model, which addresses these issues in a single model.

\subsection{Fleet Sizing}

In the effort to correctly size various types of fleets (rail car, truck, ISO, etc.), models may incorporate variability in transportation times. Seasonal peaks and dives are easily modeled in addition to vessel maintenance outages. Rail switching issues involving stoppages and congestion can also be investigated. Demand increases may be used in a current state model to determine the need for more vessels and/or how to meet the need with existing resources. ProModel has been used to meet special seasonal orders without additional resources, reduce current fleet sizes, and manage the current transportation resources more effectively.

\subsection{Inventory reduction}

Especially challenging issues in supply chain applications include capturing the behavior of continuous flow systems with stochastic conditions. On-screen variables and output statistics modules provide guidance to make decisions on tank sizing, capacity analysis and impact of variability of supply on manufacturing units. Benefits include the ability to analyze complex systems with a holistic perspective, reducing inventory levels by modeling continuous flow systems at both manufacturing and consumer sites and determining the needs of campaigning of differing products on the same unit.

\subsection{Shutdowns and outages}

The long and short-term impacts of planned outages have been a problem for managers. Unplanned and random outages are much worse. Outage issues not only include manufacturing or customer outages and their various impacts, but also involve shipping and supply outages. ProModel allows you to determine the impact of planned shutdowns on remote customer sites as well as the possibility of shutting down production because of poorly planned, customer interfaced outages. Models provide flexibility on testing alternate scenarios, moving and shifting the timing of outages as well as the duration.

\section{CONCLUSIONS}

Until recently, manufacturing companies have not fully benefited from simulation in making continuous improvements because of the time, programming expertise, and cost involved in getting useful results. ProModel is designed for manufacturing companies to fully achieve the benefits of simulation technology at an affordable price. ProModel is directed toward making simulation a standard tool in the hands of engineers, managers and systems analysts just as spreadsheet software is in the hands of accountants and financial analysts.

\section{REFERENCES}

Harrell C. R. 1990. Trends in Manufacturing Simulation. In Proceeding of Autofact Conference, eds. A. Adlard, 21-31. Detroit, Michigan.

Harrell C. R. and K. Tumay. 1992. ProModel Tutorial. In Proceedings of the Winter Simulation Conference, eds. J. Swain, D. Goldsman, R. Crain, J. Wilson, 405- 409. Arlington, VA

Harrell C. R. and J.J. Leavy. 1993. ProModel Tutorial. in Proceedings of the Winter Simulation Conference, eds. G.W. Evans, M. Moliaghasemi, E.C. Russell, W.E. Biles, 184-189. Los Angeles, CA 
Baird S.P. and J.J. Leavy. 1994. Simulation Modeling Using ProModel for Windows. In Proceedings of the Winter Simulation Conference, eds. J. D. Tew, S. Manivannan, D. A. Sadowski, A. F. Seila, 527-532. Arlington, VA

Dulgeroglu 0. and Gates G.A. 1997. Simulation to the Rescue: Stochastic Modeling Applied to Logistics. In Industrial Engineering SOLUTIONS '97 Conference Proceedings, 10-1 7. Norcross, GA.

\section{AUTHOR BIOGRAPHIES}

DEBORAH L. HEFLIN is the Director of Current Products. Her responsibilities concentrate on the development of simulation models, including consulting services, technical support and technique support. Deborah received both her B.A. in Psychology and M.B.A. in Finance from the University of Texas at San Antonio. Deborah spent 5 years working in the software manufacturing industry. Deborah has been recognized as a Texas Business Hall of Fame Scholar and a Crimson Scholar. Deborah's professional affiliations include the National Association of Female Executives, the Institute of Industrial Engineers and the Society for Health Systems.

CHARLES R. HARRELL is an associate professor of Manufacturing Engineering at Brigham Young University, and founder and chairman of PROMODEL Corporation in Orem, Utah. Dr. Harrell received his B.S. in Manufacturing Engineering Technology from Brigham Young University; M.S. in Industrial Engineering from the University of Utah; and Ph.D. in Manufacturing Engineering from the Technical University of Denmark. Prior to forming PROMODEL, he worked in simulation and systems design for Ford Motor Company and Eaton Kenway Corporation. Dr. Harrell is a senior member of IIE and SME. 